\title{
ẢNH HƯởNG CỦA RỦI RO CẢM NHÂNN ĐẾN Ý ĐINH MUA HÀNG TRỰC TUYẾN CỦA KHÁCH HÀNG TẠI TP. HỒ CHÍ MINH
}

\author{
BÙI THÀNH KHOA \\ Khoa Quản trị kinh doanh, Truờng Đại học Công nghiệp Thành phố Hồ Chí Minh; \\ buithanhkhoa@iuh.edu.vn
}

Tóm tắt: Mua sắm trực tuyến đang là một xu hướng mới của hành vi mua sắm và nó đã bùng nổ trong trong thế kỷ 21. Không thể chối cãi rằng thương mại điện tử đã mang lại nhiều lợi ích cho người tiêu dùng; tuy nhiên, vẫn còn những rủi ro trong các giao dịch trực tuyến. Nhiều nghiên cứu trước đây đã chứng minh rủi ro cảm nhận là một trong những yếu tố quan trọng ảnh hưởng đến hành vi mua hàng trong thương mại truyền thống và thương mại điện tử. Dựa trên phương pháp nghiên cứu định lượng và định tính, bài nghiền cứu đã kiểm định lại và kết luận được rủi ro cảm nhận của người tiêu dùng trực tuyến tại thành phố Hồ Chí Minh bao gồm rủi ro cảm nhận về sản phẩm/dịch vụ và rủi ro cảm nhận về quy trình giao dịch; đồng thời, rủi ro cảm nhận có ảnh hưởng nghịch biến đến ý định mua hàng trực tuyến của khách hàng. Bên cạnh đó, một số hàm ý quản trị cũng được đề xuất cho các doanh nghiệp kinh doanh trực tuyến trong nghiên cứu này để giảm các yếu tố tạo ra cảm nhận về rủi ro qua đó tăng ý định mua hàng của khách hàng.

Từ khóa: rủi ro cảm nhận, rủi ro cảm nhận về mặt sản phẩm/dịch vụ, rủi ro cảm nhận về mặt giao dịch, ý định mua hàng trực tuyến, thương mại điện tử.

\section{THE IMPACT OF PERCEIVED RISK ON THE CUSTOMER'S ONLINE PURCHASING INTENTION IN HO CHI MINH CITY}

\begin{abstract}
Online shopping is the new trend of buying behavior, and it is blooming in the $21^{\text {st }}$ century. There is no denying that the e-commerce brings many benefits for the consumers; however, there are many existing risks in online transactions. The previous researches proved that the perceived risk is one of the important factors which impact the buying behavior in both the traditional commerce and the electronic commerce. Based on the qualitative and quantitative research method, the research tests and concludes that the perceived risk based on the perception of the consumer in Hochiminh city included the product/service risk and transaction process risk, and the perceived risk will be a negative impact on the online purchasing intention of the customer. Besides that, some managerial implications for the online business are suggested in this paper to reduce the factors of perceived risk for increasing the customer's purchasing intention. Keywords: perceived risk (PR), perceived product/service risk (PRP), perceived transaction risk (PRT), online purchasing intetion (IB), e-commerce.
\end{abstract}

\section{GIỚI THIÊU}

Báo cáo của Hiệp hội Thương mại điện tử Việt Nam (VECOM) dự báo thương mại điện tử Việt Nam sẽ đạt khoảng 20 tỷ USD vào năm 2020 và tốc độ tăng trưởng trong giai đoạn 2016-2020 dự kiến sẽ đạt khoảng $20 \%$ mỗi năm. Các doanh nghiệp kinh doanh trực tuyến ra đời ngày càng nhiều, một số thương hiệu đã trở nên phổ biến và nổi tiếng như Vatgia.com, Lazada.vn, Adayroi.com, Tiki.vn, Sendo.vn; do đó, khách hàng đang dần quen với việc mua sắm trên trang web thay vì mua sắm truyền thống. Thương mại điện tử có bước phát triển nhanh chóng cùng với sự bùng nổ của hệ thống viễn thông (đển $1 / 3$ dân số truy cập Internet ở Việt Nam). Phần trăm các trang web có tính năng đặt hàng trực tuyến $58 \%$, trong khi tỷ lệ trang web có thanh toán trực tuyến là $15 \%$. Báo cáo của VECITA cũng chỉ ra những trở ngại lớn ngăn cản khách hàng tiềm năng mua sắm trực tuyến là chất lượng kém hơn quảng bá sản phẩm/dịch vụ $(73 \%)$, sau đó là vấn đề về giá cả (61\%) và dịch vụ logistics không chuyên nghiệp (45\%). Hơn nữa, trong số $38 \%$ người trả lời chưa bao giờ mua hàng trực tuyến trước đó, một nửa đã chọn "không tin tưởng vào người bán" như lời giải thích của họ, lý do tiểp theo là họ tìm sản phẩm mình muốn nhanh hơn và dễ dàng hơn trong các cửa hàng, không có thẻ tín dụng hoặc thẻ thanh toán, và lo ngại về việc tiết lộ thông tin cá nhân được lần lượt chiếm $37 \%, 26 \%$ và $25 \%$ sự lựa chọn của người trả lời [1]. Một trong những lý do đáng chú ý nhất là rào 
cản đối người tiêu dùng trực tuyến tiềm năng tham gia vào các hoạt động thương mại điện tử là những rủi ro khi mua hàng được cảm nhận bởi khách hàng [2-4]. Khi thương mại điện tử là một thị trường mới và không có đủ thông tin đáng tin cậy cũng như không đáng tin cậy, người tiêu dùng thường miễn cưỡng khi quyết định có nên mua hàng trực tuyến hay không $[1,5,6]$.

Vào năm 1960, Bauer đi tiên phong trong việc áp dụng các lý thuyết nhận thức rủi ro đến hành vi tiêu dùng và chỉ ra rằng rủi ro cảm nhận được phát triển trên cơ sở rằng tất cả các hành vi tiêu dùng liên quan đến rủi ro, trong trường hợp không chắc chắn, bao gồm những hành động được thực hiện bởi người tiêu dùng có thể tạo ra những hậu quả mà vượt quá mong đợi của họ và tạo ra sự không hài lòng [7]. Cox và Rich (1964) tiếp tục chỉ ra rằng, khi tác động tiêu cực được tạo ra bởi vì người tiêu dùng vì họ không chắc chắn về cách mua hàng để đáp ứng mức mục tiêu của họ nhiều nhất, hoặc đang lo lắng rằng việc mua hàng của họ không đạt được mục tiêu mong đợi, rủi ro cảm nhận sẽ xuất hiện [8]. Nghiên cứu chỉ ra rằng rủi ro cảm nhận kêt hợp hai khái niệm: tầm quan trọng và mức độ rủi ro từ hậu quả của việc ra quyết định bởi người tiêu dùng; và kỳ vọng chủ quan của người tiêu dùng về hậu quả tiêu cực có thể xảy ra. Hơn nữa, nếu người tiêu dùng cảm nhận xác suất sai lệch giữa kỳ vọng và các kết quả của hành động trong những tình huống do họ thực hiện hành vi thì họ sẽ nhận thấy một rủi ro không hoàn thành mục tiêu của họ tại thời điểm đó [9]. Cunningham (1967) đã đề xuất rằng người tiêu dùng cảm nhận rủi ro sẽ bao gồm mức độ nghiêm trọng của sự mất mát và kết quả bất ngờ mà có thể có thể xảy ra [10]. Nhiều nghiên cứu cho thấy rằng người tiêu dùng cảm thấy nhiều rủi ro trong các dạng mua hàng không trực tiếp tại cửa hàng; như, mua sắm qua điện thoại, mua hàng qua thư, danh mục sản phẩm [11-13]. Trong nghiên cứu của Snoj và cộng sự, niềm tin về những kết quả tiêu cực không chắc chắn tiềm ẩn từ giao dịch trực tuyến là rào cản lớn nhất cho việc kinh doanh trực tuyến của các doanh nghiệp [13]. So với các hoạt động bán lẻ truyền thống, người tiêu dùng nhận thấy mức độ rủi ro cao hơn khi mua hàng trực tuyến $[14,15]$.

Rủi ro cảm nhận là khái niệm được khai thác tương đối nhiều trong giao dịch truyền thống nhưng vẫn còn hạn chế trong các nghiên cứu thuộc lĩnh vực thương mại điện tử đặc biệt là ở Việt Nam. Hiểu được rủi ro cảm nhận của người tiêu dùng là mối quan tâm lớn đối với các nhà quản lý [16]. Mặc dù thương mại trực tuyến là một xu hướng mới trong những năm gần đây; tuy nhiên, có ít nghiên cứu tập trung vào rủi ro cảm nhận và ảnh hưởng của nó đối với ý định mua hàng trực tuyến. Do đó, nghiên cứu thực nghiệm để tìm hiểu, xác nhận về các thành phần rủi ro cảm nhận của khách hàng tại TPHCM nói riêng và Việt Nam nói chung cũng như mối quan hệ giữa rủi ro cảm nhận và ý định mua hàng trực tuyến là quan trọng và cần thiết.

\section{TỔNG QUAN NGHIÊN CÚU VÀ PHÁT TRIỂN GIẢ THUYÊTT}

Theo Solomon và cộng sự, ý định là một nhân tố thể hiện khả năng thực hiện hành vi trong tương lai của một cá nhân [17]. Ý định mua hàng trực tuyến được định nghĩa là dự tính của khách hàng sẽ dùng các kênh trực tuyến như website, mạng xã hội để mua hàng hóa, dịch vụ [18]. Trong bối cảnh của thương mại điện tử, ý định mua hàng trực tuyến được xem là tình huống khách hàng mong muốn thực hiện một giao dịch thông qua website để sở hữu được những hàng hóa dịch vụ để thỏa mãn nhu cầu của bản thân.

Rủi ro được cảm nhận trong bối cảnh mua hàng trực tuyến liên quan đến các hoạt động như mua sản phẩm, nhận thông tin không đầy đủ hoặc gây hiểu lầm; hoặc cung cấp thông tin cá nhân cho nhà cung cấp (trang web). Những hành vi này tăng rủi ro bởi vì sản phẩm có thể không chính xác như mô tả, hay trình bày trên trang web; hoặc thông tin do người bán cung cấp có thể không chính xác; hoặc thông tin cá nhân của khách hàng có thể bị sử dụng hoặc đánh cắp dẫn đến hậu quả có hại cho khách hàng [19, 20]. Rủi ro cảm nhận được định nghĩa là xác suất của khách hàng bị tổn thất tài chính do chi phí bổ sung, chi phí trong tương lai cho việc tiếp tục sử dụng sản phẩm hoặc phải tự thay thế, tự sửa chữa trong trường hợp có sự cố với sản phẩm. Bên cạnh đó, rủi ro cảm nhận còn là nhận thức rằng sản phẩm sẽ không hiệu quả như mong muốn hoặc cần thiết, khiến khách hàng bị thiệt hại do liên quan đến các lợi ích mong muốn; cũng như, mối bận tâm rằng thông tin thẻ tín dụng có thể được sử dụng theo cách bất hợp pháp (bị đánh cắp) và một số vấn đề phát sinh liên quan đến thông tin cá nhẩn của của khách hàng [21-25].

Mua hàng trực tuyến làm cho việc mua sắm bị ảnh hưởng bởi nhiều yếu tố rủi ro hơn so với mua tại các cửa hàng truyền thống [26]. Trong bối cảnh thương mại điện tử, cá nhân cảm nhận sự rủi ro trong môi trường mua sắm trực tuyến hơn so với thông thường [27]. Kannungo và Jain [28] cho rằng điều này là do thiếu sự tương tác vật lý giữa người mua và sản phẩm, trong đó gây ra một yếu tố không chắc chắn trong tâm trí của người tiêu dùng về hiệu suất dự kiến của sản phẩm. Phương thức mua sắm trực tuyến loại bỏ rất 
nhiều yếu tố quan trọng có thể được sử dụng bởi người tiêu dùng để phân tích xem một nhà bán lẻ trang web có thể được tin cậy [29]. Trong thương mại truyền thống, khách hàng có thể trực tiếp đến cửa hàng, chạm, cảm nhận, hoặc thậm chí dùng thử sản phẩm trước khi đưa ra quyết định mua hàng, trong khi người tiêu dùng trực tuyến không thể có các điều kiện như vậy. Trong khi đó, Ko và cộng sụ cho rằng người tiêu dùng cũng lo ngại về việc mua một sản phẩm từ các người bán trực tuyến; do đó, người tiêu dùng trong thương mại điện tử nhận thức rủi ro ở mức cao khi mua trực tuyến so với mua hàng truyền thống [6].

Yếu tố rủi ro liên quan đến sản phẩm/dịch vụ thể hiện sự quan ngại của khách hàng đối với việc mất tính năng, mất tài chính, tốn thời gian, mất cơ hội khi sử dụng sản phẩm/dịch vụ công nghệ thông tin [7]. Rủi ro liên quan đến sản phẩm còn được xem như khả năng chuyển giao hàng hóa không đáp ứng đúng yêu cầu như dự định ban đầu [30]. Rủi ro sản phẩm trong mua sắm trực tuyến có thể dự kiển ở mức độ cao do người mua không thể kiểm tra và thử nghiệm chất lượng sản phẩm và cũng không có sự lựa chọn để thay thế [31]. Bởi vì khách hàng không thể trực tiếp nhìn thấy, chạm vào hay dùng thử sản phẩm/dịch vụ khi thực hiện các giao dịch thương mại điện tử; do đó, sản phẩm/dịch vụ được cung cấp cho người tiêu dùng có thể không hoạt động như mong đợi [32]. Ngoài ra, người tiêu dùng có thể chịu các chi phí như vận chuyển và xử lý khi trả lại hoặc trao đổi sản phẩm/dịch vụ khi không vừa ý hoặc sản phẩm bị lỗi. Trong số năm loại rủi ro mà Jacoby và Kaplan (1972) đề xuất, kém hiệu quả và tổn thất tài chính được xem như các loại rủi ro liên quan đến sản phẩm/dịch vụ [33]. Hơn nữa, người mua hàng trực tuyến có thể lãng phí thời gian, và công sức để điều chỉnh hoặc đổi trả khi các sản phẩm/dịch vụ đã mua nếu có lỗi do nhà sản xuất hay bên bán hàng. Lãng phí thời gian là chi phí phi tiền tệ và khác nhau giữa các cá nhân, và thời gian là chi phí mà người tiêu dùng phải trả cho các sản phẩm/dịch vụ [34]. Sau khi mua sản phẩm/dịch vụ qua Internet, người tiêu dùng có thể tìm thấy một sản phẩm/dịch vụ khác có chất lượng tương đương hoặc cao hơn với mức giá thấp hơn. Do đó, khách hàng có thể mất cơ hội, đó là rủi ro khi thực hiện một hành động mà người tiêu dùng sẽ bỏ lỡ khi làm một việc khác mà họ thực sự thích làm [32].

Rủi ro liên quan đến giao dịch trực tuyển là các rủi ro có thể xảy ra khi khách hàng thực hiện giao dịch trên các phương tiện điện tử[7]. Để hoàn thành một giao dịch trực tuyến, khách hàng phải cung cấp rất nhiều thông tin cá nhân, chẳng hạn như địa chỉ, số điện thoại, và các thông tin thẻ tín dụng [35]. Người mua có cảm giác khó kiểm soát thông tin và ngần ngại cung cấp thông tin cá nhân. Nếu các thông tin cá nhân này bị mất người dùng sẽ có thể bị làm phiền, hay rủi ro về việc bị đánh cắp tiền từ các tài khoản ngân hàng hay các ví điện tử. Các nghiên cứu gần đây đã chỉ ra rằng rủi ro bị tiết lộ thông tin cá nhân, hay thông tin cá nhân bị bán cho doanh nghiệp khác trở thành mối quan tâm ngày càng tăng của người mua hàng trực tuyến [36].

Mitchell [4] cho rằng rủi ro cảm nhận là một yếu tố có ảnh hưởng mạnh mẽ trong việc giải thích hành vi mua của người tiêu dùng; bởi vì người tiêu dùng quan tâm đến việc tránh sự mất mát, thiệt hại cho bản thân họ, hơn là cố gắng đạt được những giao dịch thành công. Rủi ro cảm nhận của người tiêu dùng đã được chỉ ra là ảnh hưởng đến quyết định trực tuyến của họ [37]. Điều dễ nhận thấy là một khách hàng đang thực hiện một giao dịch trực tuyến sẽ có cảm giác rủi ro cao hơn khi so sánh với hành vi mua sắm truyền thống. Khi mua từ một cửa hàng trực tuyến, khách hàng phải cung cấp thông tin cá nhân đáng kể, bao gồm địa chỉ, số điện thoại và thậm chí cả thông tin thẻ tín dụng bí mật. Sau khi cung cấp thông tin cần thiết, người mua sắm chỉ có thể hy vọng rằng giao dịch sẽ được xử lý hoàn toàn và chính xác. Trong hầu hết các trường hợp, anh ta hoặc cô ta phải đợi hàng ngày cho đến khi sản phẩm hoặc dịch vụ được giao và giao dịch hoàn tất. Do đó, không nên ngạc nhiên rằng người tiêu dùng sẽ chú ý đến rủi ro trong giao dịch trực tuyến và rủi ro đó có thể ảnh hưởng đến quyết định của họ về việc có nên mua từ nhà cung cấp trực tuyến hay không. Ngoài ra, theo Kim và cộng sự [35], rủi ro cảm nhận của người tiêu dùng có tác động tiêu cực đối với ý định mua hàng, điều này có nghĩa là mức độ rủi ro cảm nhận càng cao, ý định mua hàng của người tiêu dùng càng ít . Hơn nữa, Chiu và cộng $s u ̛$ [38] đã tìm thấy rằng rủi ro cảm nhận của người tiêu dùng giảm ý định mua hàng trong thương mại điện tử $\mathrm{B} 2 \mathrm{C}$. Nói cách khác, rủi ro cảm nhận vẫn là một yếu tố tác động tiêu cực đến ý định mua hàng. Do đó, giả thuyết được đề xuất như sau:

\section{$\mathrm{H}_{1}$ : Rủi ro cảm nhận tác động nghich biến đối với ý định mua hàng trụ̣c tuyến của khách hàng khi mua hàng trực tuyến}




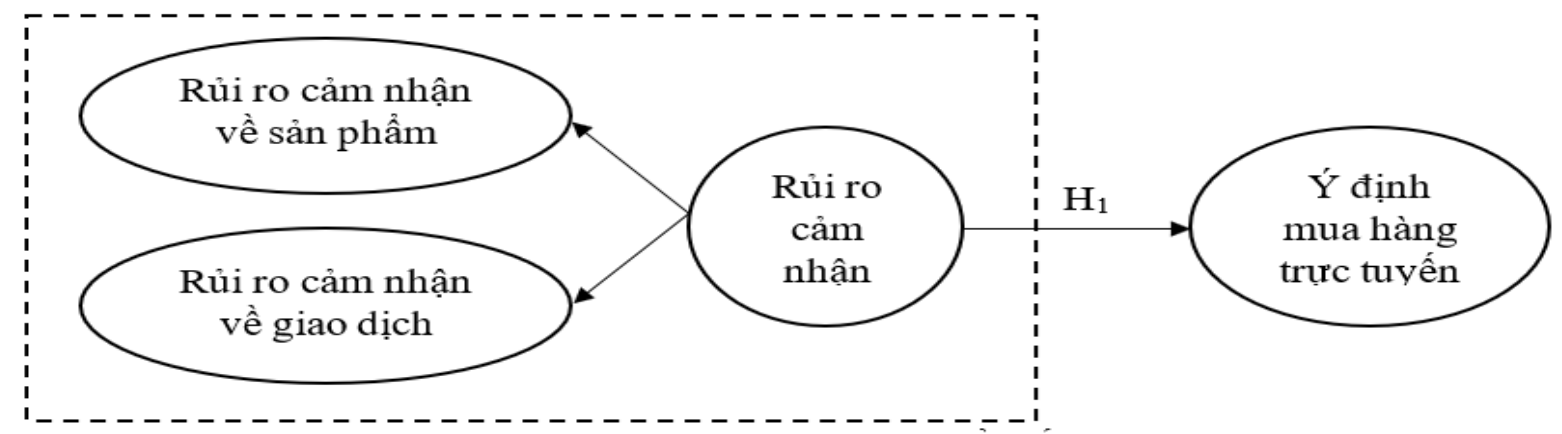

Hình 1. Mô hình nghiên cứu đề xuất

\section{PHƯƠNG PHÁP NGHIÊN CÚU}

\subsection{Phương pháp nghiên cứu và thu thập dữ liệu}

Phương pháp nghiên cứu hỗn hợp bao gồm phương pháp nghiên cứu định tính và phương pháp nghiên cứu định lượng được sử dụng để đạt được mục tiêu nghiên cứu đề ra. Nghiên cứu định tính được thực hiện nhằm tìm ra các yếu tố được xem nhu là rủi ro khi mua hàng trực tuyến cũng như dùng để điều chỉnh lại bảng câu hỏi. Phương pháp thu thập dữ liệu trong nghiên cứu định tính là thảo luận nhóm tập trung [39, 40]. Đối tượng tham gia thảo luận bao gồm 10 khách hàng được chọn bằng phương pháp phát triển mầm, có kinh nghiệm mua sắm trực tuyến trên một năm. Cuộc thảo luận được diễn ra dưới sự chủ trì của tác giả với một dàn bài hướng dẫn thảo luận. Kết quả nghiên cứu định tính cho thấy rủi ro mà 10 khách hàng nhận thấy bao gồm rủi ro về sản phẩm/dịch vụ và rủi ro trong quá trình giao dịch trực tuyến. Bên cạnh đó, tất cả người tham gia thảo luận đều nhất trí rằng rủi ro cảm nhận có tác động tiêu cực đến việc họ dự định mua hàng trực tuyến. Ngoài ra, các thang đo trong nghiên cứu về rủi ro cảm nhận cũng như ý định mua hàng trực tuyến cũng được điều chỉnh cho phù hợp với suy nghĩ của các đối tượng khảo sát. Bước tiếp theo, bảng câu hỏi được thiết kế để có thể tiến hành nghiên cứu định lượng. Đối tượng khảo sát bằng bảng câu là những tập trung vào những khách hàng có độ tuổi từ 20 đến 29 , có nghề nghiệp là học sinh - sinh viên, nhân viên văn phòng, giảng viên, vì đây là những đối tượng có nhu cầu mua sắm trực tuyến và có nhận thức về thương mại điện tử tương đối tốt [1]. Theo Hair và cộng sụ [41] thì số mẫu phải tối thiểu phải gấp 5 lần số lượng biến quan sát thì kết quả nghiên cứu mới đảm bảo tính chính xác. Tabachnick và Fidell [42] cho rằng các kích thước mẫu sẽ cho kết quả nghiên cứu tương ứng : 50 là rất kém, 100 kém, 200 là khá tốt, 300 là tốt, 500 là rất tốt và 1000 là tuyệt vời. Vì vậy, số mẫu trong cuộc nghiên cứu là 400 . Để đạt được mục tiêu nghiên cứu của đề tài, kỹ thuật chọn mẫu trong nghiên cứu này chọn mẫu phi xác suất, kỹ thuật lấy mẫu theo mục đích (judgemetal). Vì nghiên cứu đi chuyên sâu về lĩnh vực thương mại điện tử, nên áp dụng lấy mẫu theo mục đích sẽ chọn được đối tượng khảo sát hiểu rõ về vấn đề nghiên cứu và từ đó có thể mở rộng mẫu dễ dàng [43].

\subsection{Thang do}

Thang đo của tất cả các khái niệm nghiên cứu trong bài được dựa trên các nghiên cứu trước đây, được điều chỉnh qua nghiên cứu sơ bộ và thể hiện dưới dạng các phát biểu. Các thang đo sử dụng thang đo Likert với 5 mức độ từ (1) Hoàn toàn không đồng ý đến (5) Hoàn toàn đồng ý.

Bảng 1. Thống kê các nhân tố sử dụng trong nghiên cứu

\begin{tabular}{|l|c|l|}
\hline \multicolumn{1}{|c|}{ Nhóm nhân tố } & Số lượng biến & \multicolumn{1}{|c|}{ Nguồn } \\
\hline $\begin{array}{l}\text { Rủi ro cảm nhận về sản } \\
\text { phẩm/dịch vụ }\end{array}$ & 5 & $\begin{array}{l}\text { Bauer [7], Forsythe và Shi [44], Featherman và } \\
\text { Pavlou [21], Lee và cộng sụ [32] }\end{array}$ \\
\hline Rủi ro cảm nhận về giao dịch & 4 & $\begin{array}{l}\text { Sweeney và cộng sự [34], Kim và cộng sụ [45], } \\
\text { Hartono và cộng sụ [46] }\end{array}$ \\
\hline Ý định mua hàng trực tuyến & 3 & Solomon [47], Pavlou [18] \\
\hline
\end{tabular}

Nguồn: Thống kê của tác giả 


\section{3. Đặc điểm mẫu nghiên cứu}

Trong 400 đối tượng trả lời khảo sát, không có sự chênh lệch nhiều về tỷ lệ nam $(45,8 \%)$ và nữ $(54,3 \%)$. Đối tượng tham gia trả lời bảng câu hỏi tập trung là sinh viên, học sinh chiếm $65,8 \%$, đây là nhóm đối tượng thường giao dịch trực tuyến, tiếp theo là nhân viên văn phòng $(15 \%)$ và lập trình viên (11\%). Độ tuổi đối tượng khảo sát nằm trong khoảng $18-25$ chiếm $91,5 \%$ và mức thu nhập là dưới 5 triệu $(60 \%)$. Như vậy, mẫu nghiên cứu phù hợp cho mục đích nghiên cứu; từ đó sẽ góp phần giúp cho nghiên cứu có đánh giá phù hợp về ý định mua sắm trực tuyến trong mối quan hệ với rủi ro cảm nhận của khách hàng.

Bảng 2. Thông tin mẫu nghiên cứu

\begin{tabular}{|c|l|c|c|}
\hline \multicolumn{2}{|l|}{ Đặc điểm mẫu } & \multirow{2}{*}{ Số lượng } & \multirow{2}{*}{ Tỷ trọng (\%) } \\
\hline \multirow{2}{*}{ Giới tính } & Nam & 183 & 45,8 \\
\cline { 2 - 4 } & Nũ & 217 & 54,3 \\
\hline \multirow{2}{*}{ Thu nhập } & Dưới 5 triệu & 240 & 60 \\
\cline { 2 - 4 } & $5-10$ triệu & 91 & 22,8 \\
\cline { 2 - 4 } & $10-15$ triệu & 69 & 17,3 \\
\hline \multirow{2}{*}{ Nghề nghiệp } & Học sinh, sinh viên & 263 & 65,8 \\
\cline { 2 - 4 } & Giáo viên/Giảng viên & 16 & 4 \\
\cline { 2 - 4 } & Lập trình viên & 44 & 11 \\
\cline { 2 - 4 } & Buôn bán, tự doanh & 17 & 4,3 \\
\cline { 2 - 4 } & Nhân viên văn phòng & 60 & 15 \\
\hline \multirow{2}{*}{ Độ tuổi } & $18-25$ & 366 & 91,5 \\
\cline { 2 - 4 } & $26-35$ & 34 & 8,5 \\
\hline
\end{tabular}

\section{KẾT QUẢ NGHIÊN CÚU}

\subsection{Kiểm định thang đo}

Nghiên cứu này thực hiện quy trình hai bước để phân tích số liệu khảo sát thu thập được. Đầu tiên nghiên cứu sẽ phân tích nhân tố khẳng định (CFA) để đánh giá tính giá trị của các khái niệm trong nghiên cứu, sau đó sẽ thực hiện mô hình cấu trúc tuyến tính đầy đủ (SEM) để xác định mô hình đề xuất có phù hợp với mục tiêu nghiên cứu ban đầu hay không. Nghiên cứu này cũng sử dụng kiểm định Cronbach's Alpha để kiểm tra độ tin cậy các thang đo và để kiểm tra độ nhất quán nội bộ của thang đo thì tác giả sử dụng kết quả của phân tích nhân tố (EFA).

Theo bảng 3, kết quả cho thấy các thang đo đều đạt yêu cầu về độ tin cậy: Cronbach's Alpha của các thang đo đều lớn hơn 0,8 . Kết quả phân tích nhân tố (EFA - principal components with varimax rotation) cho thấy các thang đo đạt yêu cầu về trọng số $\operatorname{KMO}(>0,5)$, kiểm định Bartlett $($ sig. $>0,05)$, phương sai trích (> 50\%), Eigenvalues $(>1,0)$ và số lượng các nhân tố trích (hệ số tải nhân tố > 0,5$)$ Vì vậy các thang đo này có thể sử dụng cho nghiên cứu. Bên cạnh đó, kết quả tính toán cũng cho thấy các thang đo trong mô hình đềy đạt yêu cầu về độ tin cậy tổng hợp (C.R đều lớn hơn 0,8 ) và tổng phương sai trích (AVE đều lớn hơn 0,5$)$. 
Bảng 3. Kết quả phân tích nhân tố khẳng định cho mô hình nghiên cứu

\begin{tabular}{|c|c|c|c|c|c|}
\hline Thang đo & $\mathrm{CA}$ & FL & $\lambda$ & AVE & C.R \\
\hline Rủi ro cảm nhận về sản phẩm/dịch vụ & 0,884 & & & 0,605 & 0,884 \\
\hline $\begin{array}{l}\text { Tôi lo ngại rằng các sản phẩm sẽ không đạt hiệu } \\
\text { quả như quảng cáo. }\end{array}$ & & 0,791 & 0,855 & & \\
\hline $\begin{array}{l}\text { Tôi lo ngại rằng sản phẩm sẽ không được như tôi } \\
\text { mong đợi. }\end{array}$ & & 0,809 & 0,807 & & \\
\hline $\begin{array}{l}\text { Tôi lo ngại rằng tôi sẽ không nhận được sản } \\
\text { phẩm sau khi đã trả tiền. }\end{array}$ & & 0,791 & 0,742 & & \\
\hline $\begin{array}{l}\text { Mua hàng trên mạng khiến tôi mất đi cơ hội mua } \\
\text { hàng ở các cửa hàng truyền thống. }\end{array}$ & & 0,741 & 0,694 & & \\
\hline $\begin{array}{l}\text { Tôi lo ngại rằng sau này tôi sẽ hối hận vì đã mua } \\
\text { hàng trên trang web trực tuyến. }\end{array}$ & & 0,790 & 0,782 & & \\
\hline Rủi ro cảm nhận về giao dịch & 0,869 & & & 0,626 & 0,870 \\
\hline $\begin{array}{l}\text { Tôi lo ngại rằng trang web bán hàng thu thập quá } \\
\text { nhiều thông tin cá nhân của tôi. }\end{array}$ & & 0,791 & 0,783 & & \\
\hline $\begin{array}{l}\text { Tôi lo ngại rằng trang web bán hàng sẽ sử dụng } \\
\text { thông tin cá nhân của tôi cho các mục đích khác mà } \\
\text { không có sự cho phép của tôi. }\end{array}$ & & 0,813 & 0,808 & & \\
\hline $\begin{array}{l}\text { Tôi lo ngại rằng thông tin cá nhân của tôi không } \\
\text { được quản lý an toàn trên trang web bán hàng. }\end{array}$ & & 0,826 & 0,804 & & \\
\hline $\begin{array}{l}\text { Tôi lo ngại về các khoản phí phát sinh khi giao } \\
\text { dịch trực tuyên. }\end{array}$ & & 0,781 & 0,769 & & \\
\hline Ý định mua hàng trụ̣c tuyến & 0,834 & & & 0,629 & 0,836 \\
\hline $\begin{array}{l}\text { Tôi có suy nghĩ tích cực về việc mua sắm trực } \\
\text { tuyến. }\end{array}$ & & 0,862 & 0,779 & & \\
\hline $\begin{array}{l}\text { Có thể tôi sẽ mua sắm từ các cửa hàng trực } \\
\text { tuyến trong tương lai gần. }\end{array}$ & & 0,877 & 0,827 & & \\
\hline $\begin{array}{l}\text { Tôi có ý định mua sắm trực tuyến khi tôi cần } \\
\text { mua sắm. }\end{array}$ & & 0,853 & 0,772 & & \\
\hline $\begin{array}{l}\text { KMO } \\
\text { Sig. (Bartlett's Test of Sphericit) } \\
\text { Phương sai trích } \\
\text { Eigenvalues }\end{array}$ & & $\begin{array}{c}0,883 \\
0,000 \\
71,342 \% \\
1,273\end{array}$ & & & \\
\hline
\end{tabular}

\subsubsection{Thang đo rủi ro cảm nhận}

Như đã trình bày ở trên, rủi ro cảm nhận trong nghiên cứu này được xem như một thang đo đa hướng bao gồm hai thành phần rủi ro cảm nhận về sản phẩm/dịch vụ và rủi ro cảm nhận về giao dịch. Kết quả CFA cho thấy mô hình đo lường thang đo này phù hợp với dữ liệu thị trường với $\chi^{2}(26)=34,864(\mathrm{p}=0,115$ . 0,05$) ; \mathrm{GFI}=0,98 ; \mathrm{TLI}=0,994 ; \mathrm{CFI}=0,995$ và $\mathrm{RMSEA}=0,029$. Kết quả cho thấy các trọng số CFA đều cao $(\geq 0,694)$ và có ý nghĩa thống kê $(p<0,001$, bảng 3$)$. Vì vậy, thang đo lường các thành phần của khái niệm rủi ro cảm nhận đạt tính đơn hướng và giá trị hội tụ. Tiếp theo, hệ số tương quan giữa các thành phần của thang đo với sai lệch chuẩn kèm theo bằng $0,678(0,052)$ nhỏ hơn $1(\mathrm{p}<0.001$, bảng 4$)$. Vì vậy khái niệm rủi ro cảm nhận về sản phẩm/dịch vụ và rủi ro cảm nhận về giao dịch đạt giá trị phân biệt [48]. 


\subsubsection{Mô hình tới hạn}

Mô hình tới hạn được thành lập bằng các kết hợp giữa thang đo rủi ro cảm nhận và ý định mua hàng trực tuyến. Kết quả CFA cho thấy mô hình tới hạn phù hợp với dự liệu thị trường với $\chi^{2}(51)=58,829(\mathrm{p}=$ $0,211>0,05) ; \mathrm{GFI}=0,975 ; \mathrm{TLI}=0,996 ; \mathrm{CFI}=0,997$ và $\mathrm{RMSEA}=0,02$. Kết quả cho thấy các trọng số CFA đều cao $(\geq 0,694)$ và có ý nghĩa thống kê $(\mathrm{p}<0,001$, bảng 3$)$. Tiếp theo, hệ số tương quan giữa các thành phần của thang đo với sai lệch chuẩn kèm theo đều khác giá trị đơn vị ( $\mathrm{p}<0.001$, bảng 4$)$. Do đó, các khái niệm trong nghiên cứu đạt tính đơn hướng, giá trị hội tụ và có giá trị phân biệt giữa các khái niệm trong nghiên cứu [48].

Bảng 4. Hệ số tương quan giữa các khái niệm

\begin{tabular}{|l|l|l|c|c|c|c|}
\hline \multicolumn{2}{|c|}{ Quan hệ } & r & SE & T & p \\
\hline PRP & $<-->$ & PRT & 0,678 & 0,037 & 8,74 & 0,000 \\
\hline PRP & $<-->$ & IB & $-0,176$ & 0,049 & 23,83 & 0,000 \\
\hline PRT & $<-->$ & IB & $-0,134$ & 0,050 & 22,83 & 0,000 \\
\hline
\end{tabular}

\subsection{Kết quả mô hình cấu trúc tuyến tính}

Mô hình cấu trúc tuyến tính (SEM) được dùng đề kiểm định mô hình lý thuyết và giả thuyết. Kết quả SEM cho thấy mô hình lý thuyết phù hợp với dữ liệu thị trường với $\chi^{2}(51)=58,829(\mathrm{p}=0,211>0,05)$; $\mathrm{GFI}=0,975 ; \mathrm{TLI}=0,996 ; \mathrm{CFI}=0,997$ và $\mathrm{RMSEA}=0,02$. Bảng 5 trình bày ước lượng chưa chuẩn hóa của các tham số chính trong mô hình và hình 2 trình bày các ước lượng đã chuẩn hóa. Ghi chú là trong quá trình ước lượng các mô hình $\mathrm{CFA}$ và $\mathrm{SEM}$, hiện tượng Heywood không xuất hiện ở bất cứ mô hình nào và phương sai các sai số đều nhỏ hơn $|2,58|$.

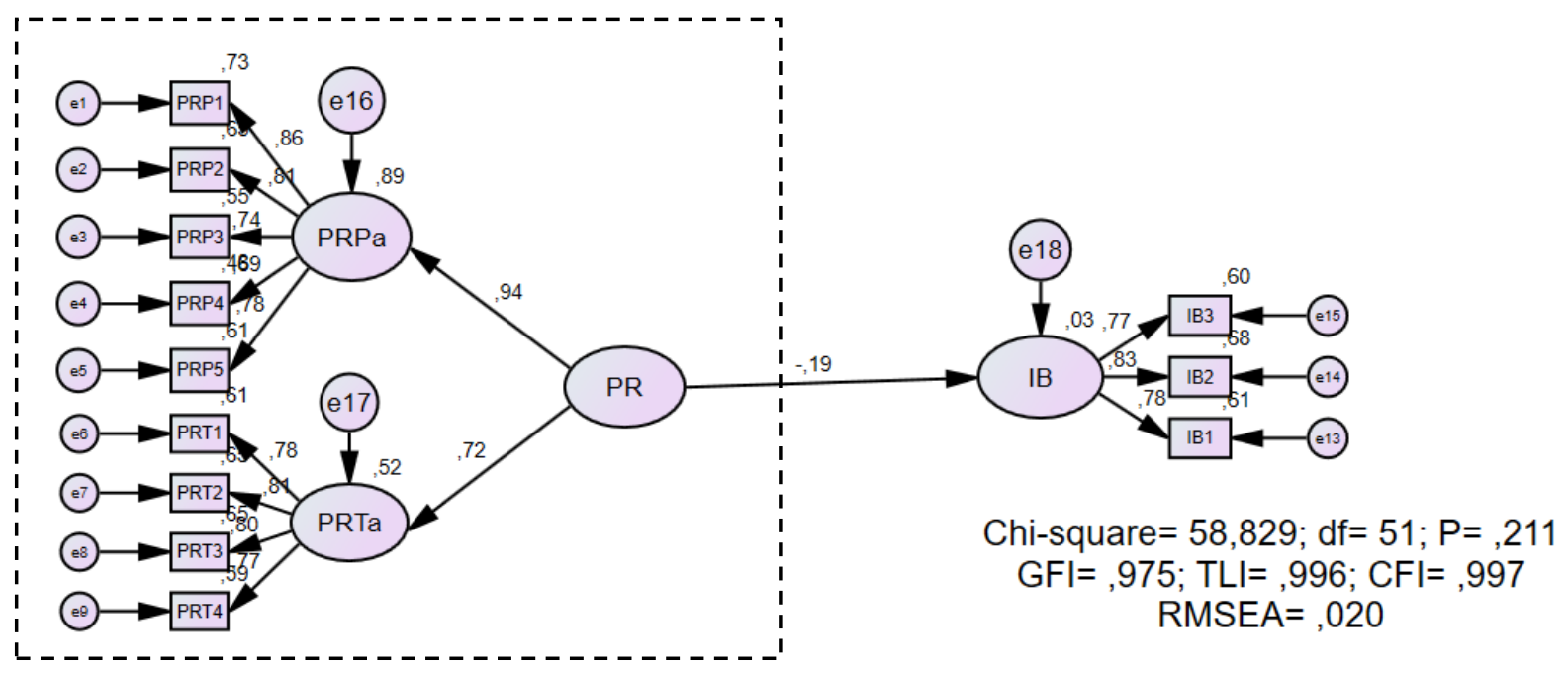

Hình 2. Kết quả mô hình cấu trúc (chuẩn hóa)

Kết quả SEM cho thấy rủi ro cảm nhận khi khách hàng giao dịch trực tuyến có mối quan hệ nghịch biến với ý định mua hàng trực tuyến $(\beta=-0,19, \mathrm{p}<0,001)$.

Bảng 5. Hệ số tương quan giữa các khái niệm

\begin{tabular}{|c|c|c|c|c|c|c|}
\hline & Quan hệ & $\beta / \gamma$ & SE & $\mathrm{T}$ & $\mathrm{p}$ & $\begin{array}{l}\text { Giả } \\
\text { thuyết }\end{array}$ \\
\hline IB & $<---$ & $-0,207$ & 0,049 & 24,61 & 0,000 & $\begin{array}{l}\text { Chấp } \\
\text { nhận }\end{array}$ \\
\hline
\end{tabular}

$\beta / \gamma$ : ước lượng, SE; sai số chuẩn, t: giá trị thống kê t(1-r), p: mức ý nghĩa 


\subsection{Kiểm định ước lượng mô hình lý thuyết bằng Bootstrap:}

Nghiên cứu này sử dụng phương pháp Bootstrap với số lượng mẫu lặp lại $\mathrm{N}=1000$. Kết quả ước lượng từ 1000 mẫu được tính trung bình kèm theo độ lệch được trình bày trong bảng 6 . Trị tuyệt đối của giá trị quan trọng $(\mathrm{C} . \mathrm{R})$ bằng $1(<2)$ nên ta có thể nói độ chệch là rất nhỏ, không có ý nghĩa thống kê với độ tin cậy $95 \%$. Vì vậy, chúng ta có thể kết luận là các ước lượng trong mô hình có thể tin cậy được.

Bảng 6. Kết quả ước lượng bằng Bootstrap với $\mathrm{N}=1000$

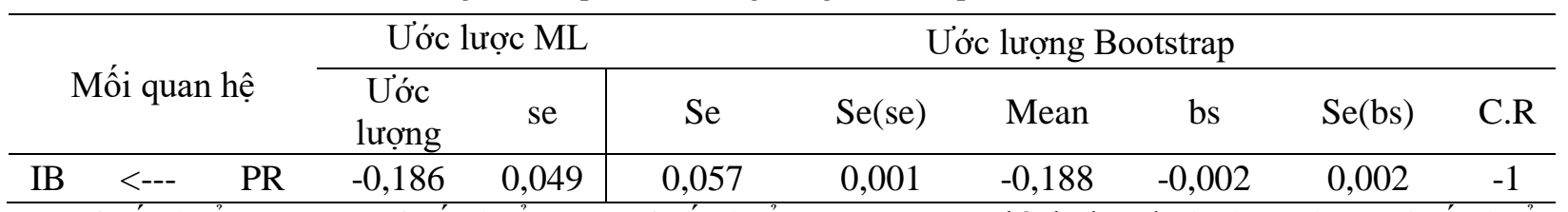

Se: sai số chuẩn; se(se): sai số chuẩn của sai số chuẩn; mean: trung bình; bs: độ lệch; se(bs): sai số chuẩn của độ lệch; C.R: giá trị quan trọng.

\section{THẢO LUẬN VÀ HÀM Ý KẾT QUẢ}

Nhận thức được tầm quan trọng của rủi ro cảm nhận và mối quan hệ giữa rủi ro cảm nhận và ý định mua hàng trực tuyển trong thương mại điện tử, nghiên cứu này kiểm định lại biến rủi ro cảm nhận dưới hình thức một khái niệm đa chiều và mối quan hệ giữa rủi ro cảm nhận và ý định mua hàng trực tuyến. Sử dụng dữ liệu thu thập từ 400 khách hàng có nhu cầu mua sắm trực tuyến tại thành phố Hồ Chí Minh, nghiên cứu đã chỉ ra được khách hàng trong thương mại điện tử cảm nhận có rủi ro về sản phẩm/dịch vụ mà họ dự định mua cũng như rủi ro có thể xảy ra trong quá trình giao dịch; hơn nữa, mối quan hệ nghịch biến giữa rủi ro cảm nhận và ý định mua hàng trực tuyến cũng là một điều cần được quan tâm bởi các doanh nghiệp kinh doanh trực tuyến. Các kết quả này mang lại một số hàm ý quan trọng cho các nhà nghiên cứu hàn lâm cũng như thực tiễn cho hoạt động quản trị bán hàng trên website.

Đầu tiên, các doanh nghiệp cần chú trọng khía cạnh sản phẩm/dịch vụ khi bán hàng trực tuyến. Để cho khách hàng có thể nhận thức được hiệu quả của sản phẩm/dịch vụ, doanh nghiệp cần cung cấp nhiều hình ảnh, video thực tế về công dụng của sản phẩm. Bên cạnh đó, cần xây dựng chức năng bình luận, phản hồi công khai dành cho khách hàng đã mua sản phẩm nhằm tạo thêm sự an tâm về hiệu quả của sản phẩm/dịch vụ. Các doanh nghiệp bán hàng trực tuyến nên trung thực trong các quảng cáo về hàng hóa/dịch vụ của mình, nên có chính sách quảng cáo đúng những lợi ích và nói ra những hạn chế của sản phẩm khi đăng bán sản phẩm trên website. Đối với các trang bán hàng lớn như Lazada, Tiki,... cần phải kiểm tra uy tín của người bán trước khi cho phép họ bán hàng trên sàn giao dịch của doanh nghiệp để luôn đảm bảo chất lượng hàng hóa đúng với mong đợi của khách hàng. Hơn nữa, cần có chức năng đánh giá người bán dành cho khách hàng từ đó sẽ tạo cho khách hàng mua mới có sự yên tâm khi mua hàng. Ngoài ra, việc tăng cường thông tin, hình ảnh, video để khách hàng có thể có nhiều thông tin hơn, tránh trường hợp chọn sai hay chọn không phù hợp. Doanh nghiệp cũng nên áp dụng hình thức "thanh toán giữ lại” bằng cách kết hợp với các doanh nghiệp chuyên về thanh toán như Visa, Marster Card hoặc Bảo Kim để tạo sự an tâm cho khách hàng về thanh toán trước khi họ nhận món hàng thực tế. Ngoài ra, các doanh nghiệp cần tạo sự khác biệt giữa mua hàng trên web và mua hàng tại các cửa hàng truyền thống; cụ thể, doanh nghiệp nên có chương trình giảm giá khi mua hàng từ website hoặc chính sách tích lũy điểm khi mua hàng từ website. Hơn nữa, các trang bán hàng trực tuyến cũng cần công bố chính sách bán hàng, đổi trả hàng hóa, chăm sóc khách hàng trên website của công ty và tư vấn khách hàng các thông tin về sản phẩm hay chính sách trước khi mua hàng qua hệ thống hỗ trợ trực tuyến.

Nhằm giảm những cảm nhận về rủi ro khi giao dịch của khách hàng, các doanh nghiệp cần công khai các chính sách về quyền riêng tư cho khách hàng được biết khi họ giao dịch, những thông tin nào sẽ được doanh nghiệp thu thập và sử dụng. Cần phải hỏi khách hàng có đồng ý cho sử dụng cookie thông qua các popup. Trước khi hoàn tất đơn hàng, nên cho khách hàng đánh dấu vào ô đã đọc và hiểu các chính sách thông tin của doanh nghiệp. Hiện tại, các trang thương mại điện tử đang sử dụng chứng nhận của Bộ Công Thương, tuy nhiên để tăng thêm độ tin cậy ở mặt quản lý an toàn thông tin cá nhân cho khách hàng, doanh nghiệp nên sử dụng "trust mark" (dấu ấn tin cậy), như TRUSTe, đó là dấu hiệu có cầu chứng các nội quy bảo vệ sự riêng tư đáng tin. Bên cạnh đó, doanh nghiệp nên sử dụng các hình thức bảo mật trong giao dịch 
như SSL (Secure Socket Layer - https://) hoặc giao thức giao dịch an toàn SET (Sercure Electronic Transaction) để đảm bảo an toàn thông tin cho khách hàng. Về chi phí giao dịch, nên có quy định rõ ràng khi khách hàng đặt mua hàng hóa, hiển thị toàn bộ chi phí khách hàng phải trả trong các giao dịch cũng như xuất hóa đơn điện tử và gửi cho khách hàng thông qua thư điện tử.

\section{HẠN CHÊ VÀ HƯớNG NGHIÊN CÚUU TIÊP THEO}

Tuy đã cố gắng thực hiện một cách khoa học và hoàn thiện nhất, nhưng nghiên cứu vẫn còn một số hạn chế. Thứ nhất, nghiên cứu chủ yếu kế thừa và ứng dụng trong lĩnh vực thương mại điện tử mô hình về rủi ro của Bauer (1960) về hai loại rủi ro cảm nhận trong môi trường kinh doanh truyền thống. Thứ hai, nghiên cứu chỉ mới thực nghiệm chung chung thương mại điện tử, mặc dù thương mại điện tử là một lĩnh vực tương đối rộng với nhiều loại mặt hàng và hình thức kinh doanh. Thứ ba, về phương pháp chọn mẫu, vì lý do thời gian và khung dữ liệu, nên tác giả chỉ có thể chọn mẫu phi xác suất thuận tiện với đối tượng khảo sát chủ yếu là sinh viên - học sinh, điều này làm giảm độ tin cậy của nghiên cứu.

Những nghiên cứu tiếp theo có thể khắc phục một số hạn chế của nghiên cứu này bằng cách tập trung vào một doanh nghiệp cụ thể hay hàng hóa cụ thể trong nghiên cứu. Bên cạnh đó, có thể xây dựng khung chọn mẫu và chọn mẫu để điều tra theo phương pháp xác suất để tạo độ tin cậy cao hơn cho nghiên cứu. Đối tượng khảo sát có thể được tăng cường như những khách hàng đã có thu nhập, việc làm ổn định, mua sắm trực tuyến bằng thẻ tín dụng để hiểu rõ hơn về rủi ro cảm nhận về giao dịch. Một số biến nghiên cứu có thể được tăng cường để có thể tạo ra nghiên cứu mở rộng, đặc biệt là các biến nghiên cứu về rủi ro cảm nhận. Cuối cùng, các biến về lợi ích cảm nhận cũng nên được nghiên cứu để đánh giá sự đánh đổi của khách hàng khi có ý định mua hàng trực tuyến.

\section{TÀI LIỆU THAM KHẢO}

[1] VECITA, Báo cáo Thương mại điện tử Việt Nam 2017, Cục Thương mại điện tử và Công nghệ thông tin, Bộ Công Thương, 2017.

[2] Y. Liebermann và S. Stashevsky, Perceived risks as barriers to Internet and e-commerce usage, Qualitative Market Research: An International Journal, vol. 5, pp. 291-300, 2002.

[3] B. Snoj, A. Pisnik Korda và D. Mumel, The relationships among perceived quality, perceived risk and perceived product value, Journal of Product \& Brand Management, vol. 13, pp. 156-167, 2004.

[4] V.-W. Mitchell, Consumer perceived risk: conceptualisations and models, European Journal of marketing, vol. 33, pp. 163-195, 1999.

[5] P. Paul, Marketing on the Internet, Journal of Consumer Marketing, vol. 13, pp. 27-39, 1996.

[6] H. Ko, J. Jung, J. Kim và S. W. Shim, Cross-cultural differences in perceived risk of online shopping, Journal of Interactive Advertising, vol. 4, pp. 20-29, 2004.

[7] R. A. Bauer, Consumer behavior as risk taking, Dynamic marketing for a changing world, vol. 398, 1960.

[8] D. F. Cox và S. U. Rich, Perceived risk and consumer decision-making: The case of telephone shopping, Journal of marketing research, pp. 32-39, 1964.

[9] J. W. Atkinson, An introduction to motivation, 1964.

[10] S. Cunningham, The major dimensions of perceived risk, Risk Taking and Information Handling in Consumer Behavior, Graduate School of Business Administration: Harvard University Press, Boston, MA, 1967.

[11] I. P. Akaah và P. K. Korgaonkar, A conjoint investigation of the relative importance of risk relievers in direct marketing, Journal of Advertising Research, vol. 28, pp. 38-44, 1988.

[12] D. Van den Poel và J. Leunis, Consumer acceptance of the Internet as a channel of distribution, Journal of Business research, vol. 45, pp. 249-256, 1999. 
[13] M. A. Eastlick và R. A. Feinberg, Shopping motives for mail catalog shopping, Journal of Business Research, vol. 45, pp. 281-290, 1999.

[14] K. S. Lee và S. J. Tan, E-retailing versus physical retailing: A theoretical model and empirical test of consumer choice, Journal of Business Research, vol. 56, pp. 877-885, 2003.

[15] M. Tan và T. S. Teo, Factors influencing the adoption of Internet banking, Journal of the AIS, vol. 1, p. 5, 2000.

[16] R. Kalakota và A. B. Whinston, Frontiers of electronic commerce vol. 19: Addison-Wesley Reading, MA, 1996.

[17] M. R. Solomon, D. W. Dahl, K. White, J. L. Zaichkowsky và R. Polegato, Consumer behavior: Buying, having, and being vol. 10: Pearson, 2014.

[18] P. A. Pavlou, Consumer acceptance of electronic commerce: Integrating trust and risk with the technology acceptance model, International journal of electronic commerce, vol. 7, pp. 101-134, 2003.

[19] D. H. McKnight và N. L. Chervany, "Trust and distrust definitions: One bite at a time," in Trust in Cybersocieties: Springer, 2001, pp. 27-54.

[20] Y.-S. Chang và S.-R. Fang, Antecedents and distinctions between online trust and distrust: Predicting high-and low-risk internet behaviors, Journal of Electronic Commerce Research, vol. 14, p. 149, 2013.

[21] M. S. Featherman và P. A. Pavlou, Predicting e-services adoption: a perceived risk facets perspective, International journal of human-computer studies, vol. 59, pp. 451-474, 2003.

[22] Z. Chen và A. J. Dubinsky, A conceptual model of perceived customer value in e-commerce: A preliminary investigation, Psychology \& Marketing, vol. 20, pp. 323-347, 2003.

[23] D. J. Brosdahl và M. Almousa, Risk perception and internet shopping: comparing United States and Saudi Arabian consumers, Journal of management and marketing research, vol. 13, p. 1, 2013.

[24] J. C. Zimmer, R. E. Arsal, M. Al-Marzouq và V. Grover, Investigating online information disclosure: Effects of information relevance, trust and risk, Information \& management, vol. 47, pp. 115-123, 2010.

[25] G. S. Milan, S. Bebber và D. Eberle, Information quality, distrust and perceived risk as antecedents of purchase intention in the online purchase context, Journal of Management Information System and E-Commerce, vol. 2, pp. 111-129, 2015.

[26] J. R. Lumpkin và M. G. Dunn, Perceived risk as a factor in store choice: an examination of inherent versus handled risk, Journal of Applied Business Research (JABR), vol. 6, pp. 104-118, 2011.

[27] G. van Noort, P. Kerkhof và B. M. Fennis, Online versus conventional shopping: Consumers' risk perception and regulatory focus, CyberPsychology \& Behavior, vol. 10, pp. 731-733, 2007.

[28] S. Kannungo và V. Jain, Relationship between risk and intention to purchase in an online context: Role of gender and product category, ECIS 2004 Proceedings, p. 95, 2004.

[29] P. A. Pavlou và D. Gefen, Building effective online marketplaces with institution-based trust, Information systems research, vol. 15, pp. 37-59, 2004.

[30] J. P. Peter và L. X. Tarpey, A comparative analysis of three consumer decision strategies, Journal of consumer research, vol. 2, pp. 29-37, 1975.

[31] E. Garbarino và M. Strahilevitz, Gender differences in the perceived risk of buying online and the effects of receiving a site recommendation, Journal of Business Research, vol. 57, pp. 768-775, 2004.

[32] D. Lee, J. Park và J.-H. Ahn, On the explanation of factors affecting e-commerce adoption, ICIS 2001 Proceedings, p. 14, 2001. 

CỦA KHÁCH HÀNG TẠI TP. HỒ CHÍ MINH

[33] J. Jacoby và L. B. Kaplan, "The components of perceived risk," in SV-Proceedings of the third annual conference of the association for consumer research, 1972.

[34] J. C. Sweeney, G. N. Soutar và L. W. Johnson, The role of perceived risk in the quality-value relationship: a study in a retail environment, Journal of retailing, vol. 75, pp. 77-105, 1999.

[35] D. J. Kim, D. L. Ferrin và H. R. Rao, Trust and satisfaction, two stepping stones for successful e-commerce relationships: A longitudinal exploration, Information systems research, vol. 20, pp. 237-257, 2009.

[36] J. Drennan, G. Sullivan và J. Previte, Privacy, risk perception, and expert online behavior: An exploratory study of household end users, Journal of Organizational and End User Computing (JOEUC), vol. 18, pp. 1-22, 2006.

[37] S. Antony, Z. Lin và B. Xu, Determinants of escrow service adoption in consumer-to-consumer online auction market: an experimental study, Decision Support Systems, vol. 42, pp. 1889-1900, 2006.

[38] C. M. Chiu, E. T. Wang, Y. H. Fang và H. Y. Huang, Understanding customers' repeat purchase intentions in B2C e-commerce: the roles of utilitarian value, hedonic value and perceived risk, Information Systems Journal, vol. 24, pp. 85-114, 2014.

[39] F. Rabiee, Focus-group interview and data analysis, Proceedings of the nutrition society, vol. 63, pp. 655-660, 2004.

[40] D. Silverman, Qualitative research: Sage, 2016.

[41] J. F. Hair, R. E. Anderson, B. J. Babin và W. C. Black, Multivariate data analysis: A global perspective vol. 7: Pearson Upper Saddle River, NJ, 2010.

[42] B. Tabachnick và L. Fidell, Multivariate analysis of variance and covariance, Using multivariate statistics, vol. 3, pp. 402-407, 2007.

[43] L. W. Neuman, Social research methods: Qualitative and quantitative approaches, 2002.

[44] S. M. Forsythe và B. Shi, Consumer patronage and risk perceptions in Internet shopping, journal of Business research, vol. 56, pp. 867-875, 2003.

[45] D. J. Kim, D. L. Ferrin và H. R. Rao, A trust-based consumer decision-making model in electronic commerce: The role of trust, perceived risk, and their antecedents, Decision support systems, vol. 44, pp. 544-564, 2008.

[46] E. Hartono, C. W. Holsapple, K.-Y. Kim, K.-S. Na và J. T. Simpson, Measuring perceived security in B2C electronic commerce website usage: A respecification and validation, Decision Support Systems, vol. 62, pp. 1121, 2014.

[47] M. R. Solomon, Consumer behavior: Buying, having, and being vol. 10: Prentice Hall Engelwood Cliffs, NJ, 2014.

[48] J.-B. E. Steenkamp và H. C. Van Trijp, The use of LISREL in validating marketing constructs, International Journal of Research in marketing, vol. 8, pp. 283-299, 1991.

Ngày nhận bài: 09/08/2018

Ngày chấp nhận đăng: 24/12/2018 